\title{
Eye Movement as a Cue to Figure Motion in Anorthoscopic Perception
}

\author{
Irvin Rock, Fred Halper, Joseph DiVita, and Deborah Wheeler \\ Rutgers University
}

\begin{abstract}
When a figure moves behind a narrow aperture in an opaque surface, if it is perceived as a figure, its shape will often appear distorted. Under such anorthoscopic conditions, the speed or direction of the object's motion is ambiguous. However, when the observer simultaneously tracks a moving target, a figure is always perceived, and its precise shape is a function of the speed or direction of tracking. The figure is seen as moving with the speed or in the direction of the target. Thus, it is argued that eye movement serves as a cue to the figure's motion, which, in turn, determines its perceived length or orientation.
\end{abstract}

When a figure is moved back and forth behind a narrow slit in an opaque surface, it is often perceived in its entirety and veridically in regard to its direction of movement. This effect has been referred to as anorthoscopic perception (Helmholtz, 1867/1962; Parks, 1965; Rock, 1981; Zöllner, 1862). ${ }^{1}$ Because only a small portion of the figure is visible at any one time, and thus apparently only a narrow column of the retina is stimulated, the effect poses a problem for any theory of form perception that assumes that an extended, simultaneous retinal image is at least a necessary stimulus correlate of perceived form.

\section{The Retinal-Painting Explanation}

One explanation of form perception under anorthoscopic conditions is that the observer's eyes move back and forth instead of remaining stationary (Anstis \& Atkinson, 1967; Fendrich \& Mack, 1981; Haber \& Nathanson, 1968; Morgan, Findlay, \& Watt, 1982; Steinbach, 1976). These eye movements are said to produce an image of the figure spread over the retina so that over time an extended image would be present. This explanation has been called retinal painting. The width of the image would depend upon the extent of eye movement so that movements equal to that of the figure would yield veridical shape perception, whereas lesser movements would lead to a compressed shape perception. In fact, just such distorted shape is often perceivd in anorthoscopic perception.

In referring to retinal painting as a possible explanation of the anorthoscopic effect, we (and other authors before us) mean

We thank Robert Fendrich for his reading of and comments on the article.

Fred Halper is now at the Department of Psychology, Essex Community College, 303 University Avenue, Newark, New Jersey 07102 . Joseph DiVita is now at the Naval Submarine Medical Research Laboratory, Naval Submarine Base, Groton, Connecticut 06349-5900. Deborah Wheeler is now at the Department of Psychology, University of Pennsylvania, 3815 Walnut Street, Philadelphia, Pennsylvania 19174.

Correspondence concerning this article should be addressed to Irvin Rock, who is now at the Department of Psychology, University of California, Berkeley, California 94720. the following. An image of the figure behind the slit is spread over the retina either because the eyes are stationary and the slit moves over the figure (the moving-slit paradigm) or because the eyes move and the slit is stationary as the figure moves behind the slit (the moving-figure paradigm). This extended image is then said to directly cause the perception of the figure in a manner very much like the typical case of form perception in which a simultaneous, extended image is present. It is presumed that by virtue of neural persistence, those parts of this extended image that had been stimulated by corresponding parts of the figure remain functionally active so that they transmit signals to the brain, much as would be the case were the figure simultaneously visible in its entirety. Whether such persistence takes the form of a positive afterimage or iconic representation has not been made explicit, although it seems to us that phenomenal representation is implicit in the retinal-painting hypothesis. The clearest example to keep in mind in thinking about this hypothesis is a rapidly moving spark or glowing cigarette seen in the dark that gives rise to the impression of an extended figure corresponding to the spark's path of motion. Those advocating this hypothesis have not dealt with the fact that in the movingfigure paradigm the figure is usually perceived to be moving. In any event, such perceived motion would not be considered relevant to the hypothesis.

\footnotetext{
${ }^{1}$ The term anorthoscopic was first used by Plateau in 1836 (cited by Helmholtz, 1867/1962) to describe a rather complex display with slits moving in front of figures and entailing distortions of shape. Later, ZölIner (1862) referred to a procedure he used of moving the figure behind a stationary slit as a new kind of anorthoscopic display. However, since then the term has come to refer to either of these procedures, the common denominator being the perception of an entire figure on the basis of successive exposure of its parts through the slit in contradistinction to the ordinary case of form perception when the entire figure is seen simultaneously. Although the term is perhaps quaint and uninformative and might better be replaced by aperture viewing as some investigators have suggested (Hochberg, 1968; Morgan et al., 1982), by now there are enough contemporary references to it to make a change in terminology inadvisable. We, therefore, use it here to mean the perception of an entire figure when it is presented successively through a narrow slit or aperture.
} 


\section{Inadequacy of the Painting Hypothesis}

There are various reasons for questioning the correctness of this explanation of anorthoscopic perception and distortion. It has been shown that an extended figure is perceived when the eyes remain stationary (Anstis, Rogers, \& Steinbach, 1976; Rock, 1981). We are referring here and in what follows (unless explicitly stated to the contrary) to the moving-figure paradigm. Unimpeachable evidence to the effect that anorthoscopic perception occurs under conditions in which an image is not spread over the retina derives from recent research using a stabilized image of the slit (and thus of whatever appears through the slit) from two different laboratories (Fendrich, 1983; Fendrich \& Mack, 1980; Morgan et al., 1982). In this technique, the subjects fixate a spot, but any slight eye movement results in the motion of the slit with the eyes. Therefore, it is now clear that retinal painting is not a necessary basis of anorthoscopic perception. Earlier research had demonstrated by eye-movement recordings that an analogous effect occurs in the moving-slit paradigm and the subject succeeds in tracking the slit (Rock \& Halper, 1969). Under these conditions too, then, retinal painting is not necessary for the perception of an extended figure (see also Rock \& Sigman, 1973; Rock \& Gilchrist, 1975).

Is retinal painting a sufficient basis of the anorthoscopic effect? There is no question that under certain circumstances when an image rapidly displaces over the retina, it leads to the impression of an extended figure. The moving spark is a case in point. Such effects can be produced in the laboratory by the moving-slit paradigm, provided that the slit moves over the figure at a sufficiently fast speed. Therefore, one might say that retinal painting is a sufficient basis of form perception via aperture viewing.

However, it does not necessarily follow that retinal painting can lead to anorthoscopic perception in the moving-figure paradigm. For that to be true, the eyes must move fast enough for the image of what is seen through the stationary slit to spread over the retina within the same time period that it does when the slit moves over a stationary figure; figure perception is achieved by such painting. The speed requirement is governed by the limits of neural persistence because one end of the figure must still be visible when the other end arrives in the aperture if it is to be seen as a simultaneous whole, in its entirety. Several investigations suggest that this value is of the order of 200 to 300 ms (Fendrich \& Mack, 1981; Haber \& Nathanson, 1968; Morgan et al., 1982). Thus, the requirement is that the eyes track a target moving at this minimum speed. If, moreover, as has been customary in anorthoscopic displays, the figure is revealed cyclically, then the eyes must move back and forth. This adds the further constraint that the eyes must stop and reverse direction. In our laboratory in unpublished research using the kind of display described below and elsewhere (Rock, 1981), subjects are simply unable to keep up with the target moving at the speed considered to be the minimum for achieving a painting effect.

In any event, until recently (Anstis \& Atkinson, 1967), investigators did not use a moving target to enable tracking, so it is unlikely that retinal painting could have been the explanation of the anorthoscopic effect reported in any of the investigations in the last century in which observers simply looked at the slit, (Helmholtz's, 1867/1962, opinion to the contrary notwithstanding). Therefore, the only question remaining as to the possible sufficiency of retinal painting as the cause of an anorthoscopic effect concerns those few laboratory studies that have used tracking. A few of these studies in which figure perception is reported meet the speed preconditions.

However, because of the nature of the phenomenal experience, there is still reason to question whether even in these rapid-speed studies the anorthoscopic effect is based on retinal painting. When a painting effect occurs at the necessary speed in the case of the moving-slit paradigm, the observer perceives the entire figure simultaneously, and it is seen within the wide region established by the image of the slit that is also spread over the retina. But this is not what is reported by Anstis and Atkinson (1967) or by Fendrich and Mack (1981) for the moving-figure paradigm (see also Fendrich, 1983). Instead, their subjects apparently perceive the extended figure but not all of it simultaneously, and the slit is seen more or less veridically as narrow. Consistent with this is the impression that the figure is moving back and forth behind the aperture, a perception that we believe is consistent with an outcome not based on retinal painting. As far as we know, there is only one report in the literature of the moving-figure paradigm in which observers perceive an extended figure simultaneously visible in its entirety wider than warranted by the size of the slit and in which the figure does not appear to be moving (Morgan et al., 1982). ${ }^{2}$

There is one further point requiring clarification. Several investigators report that at very fast speeds of the figure the impression of an extended figure is achieved only if the subjects move their eyes by tracking a moving target (Fendrich, 1983; Fendrich \& Mack, 1981; Morgan et al., 1982); free viewing will not yield figure perception under these conditions. We have also observed this fact. This in itself, however, cannot be taken as evidence for retinal painting because it may well have another explanation. At very rapid speeds the visible segments of the

\footnotetext{
${ }^{2}$ It is acknowledged by advocates of retinal painting that such perceived motion is not germane to this hypothesis, occurring for some as yet unknown reason but playing no causal role in the anorthoscopic effect (see Fendrich, 1983). In the one experiment cited earlier where retinal painting did seem to occur (Morgan et al., 1982), the figure was perceived as stationary. However, an argument that would account for this perceived motion maintains that each successively appearing element of the figure gives rise to an afterimage that is perceived to move with the eyes. Thus, the figure as a whole appears to move with the eyes. It is a known fact that afterimages do appear to move across the field as the eyes move (Mack \& Bachant, 1969). According to such a sensory account of the perceived motion, at any point in time all of the figure that has already passed behind the slit ought to be simultaneously visible and to appear to be growing in length as it moves. The slit too ought to yield an afterimage that appears to move and to increase in width as it moves. At the terminal positions of the target, the entire figure should be simultaneously visible within an equally wide, bright rectangle. But the fact is that the figure is generally not simultaneously visible and its motion is seen through the slit (for the part now in it) and amodally behind the opaque surface containing the slit (for the parts that are not now visible). An explanation of why this kind of motion of the figure is perceived is given later.
} 
figure move so rapidly within the slit in a vertical direction that they are difficult to perceive (see Fendrich, 1983). The segments may tend to mask one another, and neural persistence within the narrow vertical column may have a negative effect. Therefore, if anorthoscopic perception requires the clear perception of the position, slope, and curvature of each visible segment, as some have maintained (Rock, 1981; Rock \& Sigman, 1973), movement of the eyes may be necessary. When the eyes move, the image is spread over the retina rather than along the same retinal column, and this negative effect of rapid figure speed is eliminated. Therefore, the spreading of the image over the retina may facilitate the anorthoscopic effect, but that is by no means the same as saying that retinal painting (as defined earlier) causes the anorthoscopic effect.

Thus, if the figure is a rear-illuminated cut-out stencil viewed in the dark, but the slit itself is not visible, then the figure is not perceived even when eye movement is facilitated by introducing a rapidly moving target point. ${ }^{3}$ Under these conditions, the reported percept is that of a point of light moving up and down along a vertical axis, or because of a slight component of horizontal drift in the direction opposite tracking motion, the point of light appears to move obliquely by a few degrees off the vertical. This kind of experiment serves to remind us that the spreading of an image over the retina as a result of eye movement can ordinarily be expected to yield the perception of stationary objects that remain in a particular direction with respect to the observer (position and direction constancy). Thus, the visible part of the figure in the experiment described appears to move only vertically as the eyes move horizontally (or rather it appears to move marginally off vertical because of a slight departure from constancy). This makes it clear that the anorthoscopic effect requires something more for its occurrence, namely, information and stimulus support for the "solution" that a figure is moving behind and orthogonal to the slit. (See Rock \& Sigman, 1973). For the reasons suggested, the spreading of the image when the figure moves very rapidly can thus facilitate figure perception but not directly cause it.

Despite these facts and arguments, one finding provides elegant support for the retinal-painting hypothesis (Anstis \& Atkinson, 1967). Observers tracked a target point moving back and forth around the slit, and the speed or direction of the target was systematically varied while a circle or an ellipse was moved behind the slit. Such tracking has the effect of varying the width of the image of the figure spread over the retina or of the direction in which it is spread. One can thus make predictions of the width or of left-right orientation of the figure that should be perceived on the basis of the retinal image established; these predictions were borne out exactly by the perceptions of the observers. For example, when the target moved twice as fast as the figure, which thus produced an elongated image of the elliptical figure, observers perceived it as an ellipse whose horizontal axis was twice its objective length; when an asymmetrical target such as the letter $R$ moved left and the figure moved right, ob. servers perceived a figure that was the mirror image of the actual figure.

\section{The Construction Explanation}

We propose another explanation of anorthoscopic perception in general and of the foregoing findings in particular. Unless information about the direction of motion is provided, the perceptual system interprets the anorthoscopic pattern as an element moving in a column along the aperture. (With a vertical slit and a figure moving horizontally behind it as is typically the case, one thus tends to perceive the element moving vertically.) In fact, vertical motion is what one would predict in this situation, on the basis of Wallach's (1935) demonstration that line contours moving through an aperture will be perceived as moving in the direction of the long axis of the aperture.

Information that an entity is moving horizontally behind the slit enables the perceptual system to overcome this impression of vertical motion and to construct a figure on the basis of the temporally given information about the position of the figure's parts relative to one another. The percept is not a simultaneous one, but the impression that an entire figure is moving behind the slit is nonetheless compelling. It is a temporally extended percept and not merely a cognitive inference that a figure is behind the slit. We have found that whether or not one achieves the anorthoscopic effect depends very much on the kind of figure displayed as well as on other information such as sight of its ends. As will be seen in Experiment 4, a horizontal straight line will never yield the effect even if its ends are visible, and when the eyes are stationary, an oblique straight line will rarely, if ever, yield it, whereas a curved line that allows detection of continuous change of slope and curvature (provided the aperture slit is wide enough) usually will yield the effect (see Rock, 1981). Note that these different outcomes are not at all predictable in terms of the painting hypothesis.

The essence of this kind of explanation, in contradistinction to a retinal-painting explanation, is in the term construction. The perceptual system achieves the form percept not simply by the registration and processing of an extended retinal image but rather by integrating information over time about where the parts of the figure are in relation to one another. So, for example, suppose at Time 1 an element is visible at the top of the slit and that information is available that this element is moving to the right. If at Time 2 the element visible through the slit is lower, the system can "infer" that the two elements together form a clockwise oblique line segment. The precise slope of that line segment would depend upon the perceived speed of the elements: If it is very slow, the slope would be steep; if it is very fast, the slope would be slight. Such a constructive process can be achieved even if the eyes are stationary so that the successive visible elements stimulate only a narrow column of the retina. However, as we noted earlier, the anorthoscopic effect depends

\footnotetext{
${ }^{3}$ We believe that if the slit itself is not visible, there is no stimulus support for the anorthoscopic "solution" of a figure, most of which is occluded at any moment. It is true that investigators have shown that the slit contours revealing the figure need not be visible for an anorthoscopic effect (Fendrich, 1983; Morgan et al., 1982), but the fact is that the effect is not likely to oocur without instructions to look for it and, at that, has a long latency. Moreover, when it does occur, a phenomenal aperture with illusory contours emerges at the same time (Fendrich, 1983). That we did not obtain even this kind of effect when the slit was invisible may be explained by the kind of figure we typically use, namely, a single line, only one small part of which is visible through the slit at any moment.
} 
critically on information that the element visible in the slit is part of a larger structure that is moving behind the slit, orthogonal to it. If the slit is too narrow to allow detection of changing slope and curvature of the visible element or if the figure is a straight line or if there are no sharp discontinuities in the figure to allow detection of their actual direction of motion, then the anorthoscopic effect will probably not occur.

\section{Eye Movement as a Cue to Motion}

We have discovered a factor that will virtually guarantee the effect even for a straight oblique line and also increase the similarity of figure perception under anorthoscopic conditions to the more typical perception of an entire visible figure. When the eyes move in tracking a moving target, this immediately creates the impression that the segment seen through the slit is part of a figure moving in the direction and speed of the target. That it is the tracking of the target and not the mere presence of the moving target that produces this effect is easily proved by instructing observers to hold their eyes on the slit and not track the target.

From the standpoint of the construction type of explanation, the movement of the figure is perceived because eye movement "suggests" that one is viewing a figure moving through the narrow aperture and behind the opaque surfaces on either side, but the information registered must support or be compatible with such a perceptual interpretation. Thus, a visible element that does not move up or down the slit (see Experiment 4) will not be perceived to move, and a spot that does move vertically but is not seen within a slit will not appear to move with the eyes as they track a horizontally moving target. Note that these outcomes differ from what one would predict were the perceived motion of the figure elements based on afterimages interpreted as moving with the eyes as discussed in Footnote 2.

Thus, eye movement seems to serve as a powerful cue to figure motion without which such figure motion-and, therefore, anorthoscopic perception-often will not occur at all. Moreover, it serves as a cue as to the speed and direction of figure motion. When a figure passes behind a narrow slit, its speed and extent are ambiguous because a wider figure moving at a certain speed or a narrower figure moving at an appropriately slower speed would equally account for the appearance of its endpoints at given times. (Eye movement during the anorthoscopic presentation also seems to lead to other important effects. It can eliminate interference effects when a rapidly moving figure stimulates the same retinal column, as was noted earlier. Moreover, because it causes the image of the figure to be spread over the retina, it leads to a facilitation of the anorthoscopic percept. We return to this effect in the Discussion.)

We thus propose that the distortions of shape obtained in the experiment by Anstis and Atkinson (1967) resulted not from the extent or orientation of the image painted over the retina but from the cuing effect of eye movement on the speed or direction of figure motion. That in turn accounts for the perceived shape of the figure. The experiments reported here attempted to provide evidence for this interpretation.

\author{
Experiment 1: Effect of Varying Speed \\ and Direction of Target
}

\section{Method}

Apparatus and stimuli. In the first experiment, we presented a line figure similar to a sine curve, $0.32 \mathrm{~cm}$ thick moving behind a slit 0.12 $\mathrm{cm}$ wide ( $4.8 \mathrm{~min}$ arc) $\times 12 \mathrm{~cm}$ long $\left(8^{\circ}, 8.0 \mathrm{~min}\right.$ arc) at a speed of one pass per second. The figure was drawn in black ink on plain white paper that was affixed to a panel driven back and forth in sinusoidal motion by an arm attached to a rotating disk. The slit was cut out of black cardboard affixed to a stationary panel $1.5 \mathrm{~cm}$ in front of the moving one. A clear Plexiglas panel was positioned $1.0 \mathrm{~cm}$ in front of the screen with the slit. A luminous target $0.7 \mathrm{~cm}$ in diameter $(28.6 \mathrm{~min}$ arc) was attached to the Plexiglas panel and thus could be moved back and forth in front of the stit. This panel's speed and direction could be varied independently of the figure panel with a separate arm attached to another rotating disk. The target served as the tracking spot. Thus, the figure, slit, and target were in three separate planes: The figure was farthest from the observer, the slit was in an opaque panel in between, and the target was on a transparent panel in front. The figure panel and target panel could be moved back and forth horizontally and independently of one another while the slit remained stationary. Thus, the figure moved sideways relative to the observer and stationary slit (although the directly visible stimulus change was essentially one of vertical displacement of the parts of the figure within the slit). The target also moved relative to the observer and the slit. The slit and figure were illuminated from behind so that the figure appeared black on the bright white ground of the slit surrounded by a dark opaque region.

For judgments of the perceived length of the anorthoscopically presented figure, subjects viewed a shadow on a milk-glass screen produced by a replica of the figure drawn on glass. The glass could be rotated about a vertical axis by turning a knob. A distant light source cast a sharp shadow of the figure on the screen, the length (but not the height) of which could thus be varied by the subject.

Procedure. The observer viewed five back-and-forth excursions of the figure from a distance of $0.84 \mathrm{~m}$. There were several conditions of viewing (described below) in which each of 9 observers participated with the conditions separated from one another by at least a day.

Subjects. All 9 subjects were members of a graduate psychology department (7 students, 2 faculty members) and experienced observers of anorthoscopic perception. Three observers knew the hypothesis of the experiment but were unaware of the condition they were in at the time of participation.

\section{Results}

Free viewing. This condition served as a control. The observers were told to look at the slit while a $10.2-\mathrm{cm}$-long figure $\left(6.9^{\circ}\right)$ moved behind it. Because the figure cycled at 1 pass per second, its speed was $6.9^{\circ} / \mathrm{s}$. As expected, when the narrow slit was used, 8 of the 9 observers did not see a figure at all, and the 9th reported seeing a figure only $3.8 \mathrm{~cm}$ long. This result indicates that without the eye movements introduced in the other conditions, anorthoscopic perception does not occur. The result is predicted by the two theories but for different reasons. Because there is no extended image, retinal painting predicts no anorthoscopic effect; because there is no usable information about the figure's motion through the narrow slit and no eye movements to cue such motion, the alternative theory advocated here also predicts no anorthoscopic effect. There is simply no reason for the perceptual system to construct a percept of a moving extended figure. 
Table 1

Perceived Length of Figure Relative to Objective Length and Perceived Speed or Direction of its Motion Relative to Target Motion

\begin{tabular}{|c|c|c|c|c|c|c|c|c|c|c|c|c|c|c|c|}
\hline \multirow{2}{*}{ Experiment } & \multirow{2}{*}{$N$} & \multicolumn{4}{|c|}{ Same speed } & \multicolumn{4}{|c|}{ Double speed } & \multicolumn{4}{|c|}{ Half speed } & & \\
\hline & & Length & \multicolumn{3}{|c|}{ Speed } & Length & \multicolumn{3}{|c|}{ Speed } & Length & \multicolumn{3}{|c|}{ Speed } & \multicolumn{2}{|c|}{ Opposite direction } \\
\hline 1. Narrow slit & 9 & .77 & 8 & 1 & 0 & $\begin{array}{l}1.65 \\
1.84\end{array}$ & 15 & 0 & 0 & .50 & 6 & 2 & 1 & 9 Rev. & $8 \mathrm{Sa}$ \\
\hline $\begin{array}{l}\text { 2. Dashed line, wide slit } \\
\text { 3. Moving slit } \\
\text { 4. Control: horizontal line }\end{array}$ & $\begin{array}{l}9 \\
4 \\
4\end{array}$ & $\begin{array}{l}.97 \\
.08\end{array}$ & - & 0 & $\begin{array}{l}0 \\
-\end{array}$ & $\begin{array}{l}1.55 \\
4 \text { Rev. } \\
.11\end{array}$ & $\begin{array}{r}9 \\
4 \\
-\end{array}$ & $\begin{array}{l}0 \\
0 \\
-\end{array}$ & $\begin{array}{l}0 \\
0 \\
-\end{array}$ & .55 & 9 & 0 & 0 & $\begin{array}{l}9 \mathrm{NF} \\
4 \mathrm{El} .\end{array}$ & $\overline{4 \mathrm{Sa}}$ \\
\hline
\end{tabular}

Note. For the free-viewing condition: Experiment 1, no figure was perceived by 8 of the 9 subjects; Experiment 2 , length $=1.02$. NF $=$ no figure perceived; $E=$ equal; $F=$ faster than; $S$ = slower than; $E I .=$ elongated; $S a$. = same; Rev. = reversed. The speed, length, orientation, and direction are as perceived by the subject. The dashes in cells indicate that speed or direction data were not obtained because a moving figure was not perceived. Empty cells indicate that the condition was not included.

a $N$ 6. This result refers to the 6 additional subjects who viewed a smaller figure; thus the entry under Speed is 15 instead of 9.

Target motion twice that of figure. In another condition, the observers tracked the luminous target, which moved back and forth in front of the slit at twice the speed of the figure. The velocity of the figure was $13.8^{\circ} / \mathrm{s}$. When a $10.2-\mathrm{cm}$ figure was displayed, the average judgment of its length was $16.8 \mathrm{~cm}$. In Table 1 the results for perceived length are given relative to objective figure length. That ratio was 1.65 .

Because in this condition the target necessarily moved far from the slit, the ends of the rather long figure were visible only by quite peripheral vision $\left(7^{\circ}\right)$. Therefore, the condition was repeated with 6 subjects and a smaller, $5.1-\mathrm{cm}-$ long $\left(3.4^{\circ}\right)$ figure (velocity: $6.8^{\circ} / \mathrm{s}$ ). In this case the average match of its length was $9.4 \mathrm{~cm}$, which, relative to the true length of $5.1 \mathrm{~cm}$, was 1.84 . This perceived elongation of almost twice the figure's length can in principle be explained by the retinal-painting hypothesis. However, we also required the observers to judge the perceived speed of the figure in relation to the tracked target by indicating whether it appeared to be moving faster than, slower than, or at the same speed as the target. Of the 15 observers, all reported the speed to be the same or approximately the same as that of the target. Table 1 gives data on perceived speed as well as on perceived length ratio of the figure for this and all other experi. ments. This finding concerning perceived speed is not relevant to the retinal-painting hypothesis for the simple reason that the image spread over the retina is considered to be the necessary and sufficient explanation of the anorthoscopic perception; perceived motion of the figure either is not predicted or is considered epiphenomenal. However, both the perceived motion and perceived length follow from the eye-movement-cuing hypothesis. To be specific, given the "belief" (based on eye-movement cuing) that a figure is moving behind the slit and that it is doing so at the speed the eyes are moving, the perceptual system will construct a percept of a figure that is roughly twice the objective length of the stimulus figure. That is, if the figure's left end arrives in the slit at Time 1, if it is moving to the left at the rate of $\theta^{\circ} / \mathrm{s}$, and if its right end arrives in the slit at Time 2 , then in the $I \mathrm{~s}$ between Time 1 and Time 2 (the speed for one pass) the figure can be inferred to be $\theta^{*}$ long. $\theta$ here is given by the regis- tered speed of eye movement during smooth pursuit of the target.

Target motion half that of figure motion and equal to that of figure motion. In another condition, the target moved at half the speed of the figure (velocity $=3.4^{\circ} / \mathrm{s}$ ). The average match of the figure's length for the 9 observers was exactly half of its actual length (ratio of .50). Of these 9 observers, 6 said that the figure's speed was equal to that of the target, 1 that it was slower, and 2 that it was faster. In still another condition, the target moved at the same speed as the figure. The average match in this case was .77 of the objective length. Of the 9 observers, 8 reported the figure was moving at the same speed as the target and $I$ as moving faster. Here again, both theories predict the length of the figure reported in the two conditions, but only the construction theory predicts the perception of the perceived speed in these two conditions.

Target motion opposite direction of figure motion. In this final condition, a figure moved in one direction while the target moved the same distance and speed in the opposite direction (in antiphase). The focus of interest here was in the perceived orientation of the figure as well as in its perceived direction of motion. All 9 observers perceived the mirror image of the actual figure as was ascertained by a sketch they were required to make. This much is predictable in terms of the reversal of the retinal image. However, 8 observers reported the direction of the figure's motion to be the same as that of the target, which, of course, was incorrect. ${ }^{4}$ Thus, the phenomenal reversal of figure orientation is explicable in terms of the misperception of direction of figure motion, which, in turn, is explicable in terms of

\footnotetext{
- One subject reported that the figure appeared to move in a direction opposite to that of the target, which was in fact the case. Such a perception is not compatible with the perception of the mirror-image shape according to our hypothesis. However, this same subject reported such opposite direction of figure motion in the conditions (described earlier) where target and figure move in the same direction, a perception which no other subject ever reported and for which there is no plausible explanation. Therefore, we feel we are justified in discounting this subject's deviant report in the condition under discussion.
} 
the cuing effect of eye movement. To be specific, if the perceptual system "assumes" (in this case incorrectly) that a figure is moving, say, leftward, then the orientation of the figure constructed will be based on this assumption together with the time of arrival within the slit of the elements of the figure. For example, if the top arrives first and the bottom last, then the figure must be one that is tilted counterclockwise. But for rightward movement, the figure would have to be tilted clockwise.

\section{Experiment 2: Effects of Introducing Good Horizontal Direction Information}

\section{Method}

In a variation of this experiment, a dashed-line figure instead of a solid-line figure was used, and the slit was widened to $0.48 \mathrm{~cm}(19.8$ $\min$ arc). These changes were expected to provide good information concerning the direction or speed of figure motion. One can now readily detect that the endpoints of each dash are moving left or right, whereas with the continuous solid line and narrow slit used in Experiment 1 such information is not available. Indeed, now in a free-viewing condition, all 9 observers perceived a figure, and their average match of its length was very close to that of the actual figure. The question posed was whether this direct information concerning the motion of the figure would overpower or be overpowered by the eye-cuing effect in those conditions where a conflict existed.

\section{Results}

The result was that with figure and target moving at the same speed, the average match was 0.97 of the actual length. All of these observers reported the figure's speed to be the same as that of the target. When, however, the target moved at either half the speed or twice the speed of the figure, the eye-cuing effect was strong enough to cause an appreciable distortion. The average relative length match was 0.55 in the first case and 1.55 in the second case. Moreover, all 9 observers in both cases reported the figure to be moving at the same speed as the target despite the potentially good information as to its actual speed. Of special interest in this experiment was the condition in which figure and target moved in antiphase. Not a single observer perceived a figure. Subjects reported seeing a small flickering element moving up and down the slit. We interpret this to mean that a conflict existed. The information through the slit as to the direction of figure motion and the effect of eye-movement cuing opposed each other so that no figure motion at all was perceived. Consequently, anorthoscopic perception did not occur. This condition poses particular difficulties for the retinal-painting hypothesis because the image of the figure would be spread over the retina in reversed direction just as in the corresponding condition of the previous experiment and, thus, should lead to anorthoscopic perception with left-right reversal.

\section{Experiment 3: Moving Slit Variation}

\section{Method}

The power of the effect of eye movement as a cue to figure motion was brought out in another experiment in which we reversed the usual procedure by moving the slit over a stationary figure. The target moved with the slit, in phase with it, but at twice its speed. We had intended this experiment as a control because we expected the figure to be perceived veridically, nonreversed and stationary - which it always is when the observer simply looks at the moving slit and no moving target is present-despite the reversal of the retinal image produced by the moving target.

\section{Results}

The result, however, was that the figure was incorrectly perceived as moving behind the slit by all of 4 observers in the direction and at the speed of the target, with the consequence that it was seen as left-right reversed. In a variation of this procedure, the target moved in antiphase to the slit but at the same speed, again with the figure stationary. Here the figure appeared very elongated and seemed to be moving in the same direction as the target. So in this experiment the perceived orientation or length of the figure is again predictable on the basis of either the image painted over the retina or of the figure's perceived direction and speed of motion. However, once again, the retinalpainting theory does not consider the perception of figure motion to be relevant.

\section{Experiment 4: Horizontal Line Figure Control}

\section{Method}

As a final control for the possibility that all of our results concerning perceived distortion are explicable on the basis of the image painted over the retina and that the results concerning perceived motion of the figure are somehow irrelevant, we moved a $10.2-\mathrm{cm}$-long horizontal straight line behind the slit. The target moved at either the same speed or twice the speed of the figure. Because such a figure does not displace up or down the slit, there is no motion visible whatsoever, only a stationary display, and, therefore, there is no reason to expect eye movement to cue figure motion. The situation is much like the one that occurs in daily life when one views a stationary object while tracking another object. Yet an image of a horizontal line is painted over the retina, and all conditions of stimulation, such as speeds, luminance, and contrast, are the same here as in Experiment 1.

\section{Results}

The results for 4 observers were that none perceived an extended figure, and none perceived anything like figure motion. There was a tendency to perceive the line as slightly longer than the segment that was visible at any moment, the average for the same-speed condition being $0.80 \mathrm{~cm}$. (32.7 $\mathrm{min}$ arc) and for the double-speed condition, $1.1 \mathrm{~cm}(45 \mathrm{~min}$ arc). The slit also seemed to be slightly wider than its actual width, at least for brief moments during the tracking. These slight enlargements can be attributed to a partial retinal-painting effect, but they are qualitatively and quantitatively totally different from the anorthoscopic perception that occurred in the previous experiments. Qualitatively, there is no impression of a figure moving behind the slit; quantitatively, the length perceived is only $1 /$ 10th of the figure's objective length.

\section{Discussion}

Despite all these arguments and evidence, one might remain unconvinced that retinal painting is not the basis of the findings 
reported here. After all, in almost every case, what is perceived is perfectly correlated with the retinal image of the figure established: whether no extended figure is perceived (Experiment 1, free viewing); whether the figure is perceived as compressed, actual length, or elongated (Experiment 1); whether the figure is perceived as left-right reversed or not (Experiment 1 and Experiment 3). Admittedly, this is formidable evidence, but most of it was available earlier (Anstis \& Atkinson, 1967), and we have attempted to offer a different account of why there occurs such a correlation between image established and figure perceived. However, as we mentioned earlier, we now know with certainty that retinal painting is not a necessary condition for the anorthoscopic effect. One can achieve the effect in viewing a stationary slit with eyes stationary even though under the particular conditions of free viewing in Experiment 1 no such effect was obtained. (Note, however, that it was obtained in a freeviewing variation of Experiment 2.) Moreover, we have now also seen that retinal painting is not a sufficient basis of the anorthoscopic effect. It does not occur in the antiphase condition of Experiment 2 and in Experiment 4. Therefore, all these facts must be taken into account in assessing the meaning of the experiments we describe here.

Can the findings concerning perceived speed and direction of figure motion be explained in a way that would be consistent with the retinal-painting hypothesis? Suppose the perceived shape of the figure is the cause rather than, as we have argued, the effect of the perceived speed and direction of motion. Suppose that the perceived shape were to derive directly from the image painted over the retina as Anstis and Atkinson (1967) maintained. Given a particular perceived shape, the observer might then infer its speed accordingly. For example, an elongated figure would have to be moving much faster than a compressed one to account for the same time of arrival in the slit of its endpoints. Similarly, a clockwise oblique line figure moving leftward that appeared counterclockwise oblique could be inferred to be moving rightward if its bottom end appeared at the beginning and its top end at the termination of its motion.

The difficulties with this argument can be summarized as follows: (a) It is based on the presupposition that the anorthoscopic effect of an extended phenomenal shape in our experiments derives from retinal painting although the speed we used of one pass per second is far too slow to achieve such an effect: Experiment 4 shows directly that no such effect occurs under our experimental conditions. (b) As we noted earlier, if the perceived figure is the result of retinal painting, it ought to appear as a simultaneously given whole, not as a moving figure, only part of which is visible at a time. On the other hand, the impression of a figure in motion, the parts of which are seen only successively, is at the very heart of our explanation of the effect. (c) The impression of a figure moving at the predicted speed or direction occurs from the very outset, whereas according to the foregoing explanation such speed or direction could be inferred only after the figure's shape was perceived.

Fendrich (1983) has sought to test the eye-movement-cuing hypothesis by creating conditions in which such cuing would be separated from retinal painting. Subjects tracked a moving target as in the experiments reported here, but the image of the slit was stabilized by moving it in tandem with the eyes. This procedure failed to yield the kind of effect reported here. For example, figure perception was no better under such conditions than when the subject was required to fixate a stationary target; figure perception was not left-right reversed if a target moved in one direction and the figure moved in the opposite direction when stabilization was used to move the slit with the eyes. Unfortunately, however, this technique is not suitable for testing the hypothesis, because what the hypothesis maintains is that the figure seen through the slit appears to move with respect to it, at the speed or in the direction that the eyes move with respect to it. If the slit is constrained to remain in the same position relative to the target as in Fendrich's method, then there is no reason to predict that the figure will be cued to move across it at or at least to do so by virtue of what the eyes are doing. Thus, for a proper test of the hypothesis, the eyes must be moving with respect to the slit.

Some results reported by Morgan et al. (1982) may be explicable in terms of the eye-cuing hypothesis rather than, as those authors believe, in terms of retinal painting. They used the same method that Fendrich (1983) did, namely, one in which subjects tracked a moving target while viewing a figure through a slit, the motion of which was stabilized, only partially stabilized, or not stabilized at all. When they sought to directly test the effect of degree of retinal painting on the perceived width of the figure presented, they found that the perceived width of the figure did correlate significantly with the width of the image spread over the retina by virtue of eye movements: By and large, perceived figure width was maximum with no stabilization and minimum with perfect stabilization. Reinterpreting this finding in terms of our comments in the previous paragraph, these results may mean that a strong eye-cuing effect occurred with no stabilization, so that the figure appeared to move across the slit as much as the target moved, and that no such cuing effect occurred with stabilization, so that the figure did not appear to move much, if at all (unless the aperture was relatively wide and the speed of figure motion relatively slow). With rapid speed and narrow aperture, conditions are poor for the anorthoscopic effect unless facilitation is provided by eye movement in the manner suggested here. ${ }^{5}$

The evidence would thus seem to rule out retinal painting as the basis for the anorthoscopic perception and the specific distortions obtained in our experiments. Yet the effects obtained here are vivid, sensory-like shape percepts. These effects are no doubt at least partially based on the fact that the image is spread over the retina because the percept obtained under the conditions of tracking used is "better" than the anorthoscopic effect obtained when the eyes remain stationary. Also, we found a similar facilitation of anorthoscopic perception when, in the moving-slit paradigm, the eyes are held stationary rather than tracking the slit. In this situation cuing via eye movement is not a factor. Thus, we believe two factors explain our results in the moving-figure paradigm: the cuing of figure motion by eye

\footnotetext{
${ }^{5}$ Of course, if these subjects experienced the entire figure simultaneously and did not have any impression of figure motion as was the case in Morgan et al.'s (1982) Experiment 1 described earlier, then our speculations here about an eye-movement cuing rather than a retinalpainting explanation would be incorrect.
} 
movement and the facilitation of anorthoscopic perception by the presence of an image spread over the retina.

It may seem confusing to say that an extended image of the figure can facilitate the anorthoscopic effect but yet deny that this outcome is based on retinal painting. Referring to the definition of retinal painting given earlier, we argue that the anorthoscopic percept is not a simultaneous one and the presence of the extended image is neither a necessary nor a sufficient cause of the percept as it should be if retinal painting were the explanation. To repeat, it is not necessary because the effect occurs in the absence of any extended image (Fendrich, 1983; Fendrich \& Mack, 1980; Morgan et al., 1982), and it is not sufficient for a variety of reasons (see Rock, 1981), including the fact that no such effect occurs when a horizontal line is exposed (as in Experiment 4). Therefore, we believe that anorthoscopic perception requires a constructive process of inferring shape from successive views of the parts of the figure interpreted by the perceptual system as successive adjacent spatial regions of the figure. Apparently, this process is facilitated when the successive views are spread out appropriately over the retina. In the moving-figure paradigm, however, the facilitation does not occur in the absence of the eye-movement-cuing effect. The evidence suggests that the perceptual system constructs a figure of a certain length and orientation based on the "suggestion" of the moving eyes that a structure is moving behind the slit at a certain speed and direction.

What is not yet entirely clear is the explanation of the tendency of eye movement to serve as a cue to figure motion. This tendency is broader than its application to the anorthoscopic paradigm. We suggest that eye movement plays this cuing role whenever a display is ambiguous as to the specifics of its motion. The speed and direction of motion of the element or elements within a narrow slit are ambiguous, but the slit itself, being continuously visible, is, of course, unambiguously stationary and thus does not partake in the cued motion of the elements within the slit. There is evidence of such an effect in certain other situations. When oblique lines are viewed moving behind a square aperture, the perceived direction of line motion is ambiguous and often reverses (Wallach, 1935). If a target is introduced, moving in phase with what the lines' horizontal or vertical displacement would be if they were actually moving in one of these directions, we have found that the lines will almost always appear to move in the direction of that target, provided that the observer tracks it. Thus, the experimenter can easily induce the observer to reverse by the simple expedient of changing the path of the target. ${ }^{6}$

Another instance in which eye movement influences the direction of perceived motion occurs in the kinds of display studied by Johansson (1950). For example, in his well-known demonstration in which two points move along the paths of the arms of an $\mathrm{L}$, they tend to appear to move apart or together along an oblique path rather than (veridically) along paths at right angles to one another. However, if one point is deliberately tracked, it tends to be seen veridically as moving either horizontally or vertically. Presumably, then, the direction of eye movement serves as a cue to veridical perception that otherwise, in this example, would not occur. Still another instance in which eye movement can affect motion perception is with ambiguous ap- parent motion displays. If a multiple-dot display can appear to oscillate or to move continuously clockwise or counterclockwise, then instructions to move the eyes in one of these alternative sequences will bias the outcome accordingly. Ward and Morgan (1978) reported that dots in a visual noise display will appear to move along with pursuit eye movements, a kind of entraining effect. The common denominator in these various cases would seem to be an "assumption" on the part of the perceptual system that the eyes are tracking the moving object or an object keeping pace with the moving one. Therefore, the direction or speed of eye movement informs about the direction or speed of the object's motion.

In the experiments reported here, we have examined only the distortion of anorthoscopic perception that occurs on the basis of eye movement. But distortion of shape also occurs in the more typical experiment where tracking of a target is not introduced. Under conditions of free viewing, particularly when the figure moves rapidly, the shape perceived is often compressed along its axis of motion. Distortions also occur when the slit is curved or slanted away from rather than orthogonal to the direction of figure motion (Anstis \& Atkinson, 1967; Hecht, 1924). One might seek to explain such distortion directly in terms of the retinal image produced on the basis of eye movements, but we believe it is based upon the misperception of the figure's speed, which is most typically an underestimation. Because information about figure speed from the vertical displacement of its contour through the slit is poor, it would not be surprising if its speed were misperceived. Underestimation may be a conservative tendency under such conditions. Moreover, if perceived speed increases very little or not at all as actual figure speed is increased, we should expect that the distortion would increase with figure speed, as seems to be the case. We have shown in an as yet unpublished study that figure speed is indeed underestimated under free-viewing anorthoscopic conditions (Rock \& DiVita, 1981). Given underestimation, form construction based on perceived speed should predictably lead to a compression effect, and slanted apertures should yield the distortions they do because the region of the figure crossing the aperture first would not appear to be as far from the region crossing it last as it actually is.

\footnotetext{
${ }^{6}$ It has been suggested to us by Arien Mack (personal communication, 1985) that this effect, the tendency to perceive the lines moving in the direction of a tracked target, is explicable in terms of the more general principle that images that remain stationary on the retina will appear to move with the eyes. If this principle can indeed be extended to account for this effect, then although eye movement would play a crucial role in determining the perceived direction of motion of the visible contours, it would do so somewhat more automatically than we have suggested by our use of the word cue. Stationary images on the retina appear to move because the perceptual system lawfully integrates information about retinal locus and eye position in achieving perception of radial (or egocentric) direction as in position and direction constancy. The difficulty with this argument, however, is the question of whether it is correct to say that the images of the moving lines are indeed stationary during tracking when their direction of motion over the retina can be regarded as ambiguous.
} 


\section{References}

Anstis, S. M., \& Atkinson, J. (1967). Distortions in moving figures viewed through a stationary slit. American Journal of Psychology, 80 , 572-585.

Anstis, S. M., Rogers, G., \& Steinbach, M. (1976). [Research on anorthoscopic perception]. Unpublished raw data, York University.

Fendrich, R. (1983). Anorthoscopic figure perception: The role of retinal painting produced by observer eye motion. Unpublished doctoral dissertation, New School for Social Research.

Fendrich, R., \& Mack, A. (1980). Anorthoscopic perception occurs with a retinally stabilized stimulus. Supplement to Investigative $O p h$ thalmology and Visual Science, 19 (Suppl.), 166.

Fendrich, R., \& Mack, A. (1981). Retinal and post-retinal factors in anorthoscopic perception. Supplement to Investigative Ophthalmology and Visual Science, 20(Suppl. 3), 17.

Haber, R. N., \& Nathanson, L. S. (1968). Post-retinal storage? Some further observations on Parks' camel as seen through the eye of a needle. Perception \& Psychophysics, 3, 349-355.

Hecht, H. (1924). Die simultane Erfassung der Figuren [On simultaneous apprehension of figures]. Zeitschrift fur Psychologie, 94, 153194.

Helmholtz, H. von (1962). Handbook of physiological optics (3rd ed., J. P. C. Southall, Trans.). New York: Dover. (Original work published 1867)

Hochberg, J. (1968). In the mind's eye. In R. N. Haber (Ed.), Contemporary theory and research in visual perception (pp. 309-331). New York: Holt, Rinehart \& Winston.

Johansson, G. (1950). Configurations in event perception. Uppsala, Sweden: Almkvist \& Wiksell.
Mack, A., \& Bachant, J. (1969). Perceived movement of the after-image during eye movements. Perception \& Psychophysics, 6, 379-384.

Morgan, M. J., Findlay, J. M., \& Watt, R. J. (1982). Aperture viewing: A review and a synthesis. Quarterly Journal of Experimental Psychology, 34A, 211-233.

Parks, T. (1965). Post-retinal visual storage. American Journal of Psychology, 78, 145-147.

Rock, I. (1981). Anorthoscopic perception. Scientific American, 244, 145-153.

Rock, I., \& DiVita, J. (1981). Underestimation of speed in anorthoscopic perception. Unpublished manuscript.

Rock, I., \& Gilchrist, A. (1975). Induced form. American Journal of Psychology, 88, 475-482.

Rock, I., \& Halper, F. (1969). Form perception without a retinal image. American Journal of Psychology, 82, 425-440.

Rock, 1., \& Sigman, E. (1973). Intelligence factors in the perception of form through a moving slit. Perception, 2, 357-369.

Steinbach, M. (1976). Pursuing the perceptual rather than the retinal stimulus. Vision Research, 16, 1371-1376.

Wallach, H. (1935). Über visuelle wahrgenommene Bewegungsrichtung [On the direction of motion of straight lines]. Psychologische Forschung, 20, 325-380.

Ward, R., \& Morgan, M. J. (1978). Perceptual effect of pursuit eye movements in the absence of a target. Nature, 274, 158-159.

Zöllner, F. (1862). Über eine neue Art anorthoskopischer Zerrbilder [A new kind of anorthoscopic display]. Annalen der Physic, 117, 477484.

Received February 18, 1986

Revision received October 17, 1986

Accepted January 26, 1987

\title{
Cutting Appointed Editor, 1989-1994
}

The Publications and Communications Board of the American Psychological Association announces the appointment of James E. Cutting, Cornell University, as editor of the Journal of Experimental Psychology: Human Perception and Performance for a 6-year term beginning in 1989. The current editor, William Epstein, will be receiving submissions through September 30,1987 . At that point, the 1988 volume will have been filled, and all submissions after that should be sent to James Cutting. Therefore, as of October 1, 1987, manuscripts should be directed to:

\author{
James E. Cutting \\ Department of Psychology \\ Uris Hall \\ Cornell University \\ Ithaca, New York 14853-7601
}

